\title{
Evaluation of OTT PLUVIO Precipitation Gage versus Belfort Universal Precipitation Gage 5-780-Supplemental Data, January 15 through July 16, 2002
}

\section{Background}

The National Atmospheric Deposition Program (NADP) was established in 1977 to study atmospheric deposition and its impact on the environment. The program's National Trends Network (NTN) includes wet atmospheric deposition networks at more than 250 sites across the United States, Canada, Puerto Rico, and the Virgin Islands (National Atmospheric Deposition Program, 2003). Precipitation amounts are currently measured at all NTN sites using the Belfort Universal Precipitation Gage 5-780 (Belfort), which involves technology that is more than 50 years old.

In 1999, a three-phase study was begun to evaluate several weighing, all-weather precipitation gages to find a possible replacement for the Belfort gage. One gage that performed consistently well in phase I and II of the study was the OTT PLUVIO precipitation gage (OP). Phase III of the study was to determine the accuracy and comparability of the precipitation data collected using the OP gages and the existing Belfort gages. The NovaLynx Model 260-2510 Standard Rain and Snow Gage (NovaLynx) was used as a reference at two sites. Seven OP gages were installed at six NTN sites across the country, representing a broad range of climatic regimes, for a data collection period of 18 months. The six test sites were: Sand Mountain, Ala. (AL99), Bondville, Ill. (IL11 West and East), Marcell, Minn. (MN16), Smith Valley, Nev. (NV03), Penn State, Pa. (PA15), and Brooklyn Lake, Wyo. (WY95). A report describing the phase III precipitation gage evaluation is available by accessing $<$ http://pubs.water.usgs.gov/wri034167>. After the phase III data collection period ended the NADP extended the data collection another six months, from January 15 through July 16, 2002. This fact sheet supplements the phase III report and evaluates the additional six months of data collected.

For the additional six months of data collection the OP gages at IL11 East and West, MN16, NV03, and PA15 were still in operation. As reported in the original report (Tumbusch, 2003), data-retrieval difficulties caused sites AL99 and WY95 to end participation in the study before the original 18-month data-collection period ended.

Data loss varied considerably from gage to gage (table 1).

Mechanical difficulties were a major problem with the Belfort gages; the OP gages lost data because of downloading problems. The OP gage at PA15 had no lost data, while the corresponding Belfort gage lost 28 days out of 183 data collection days.

\section{Results}

A summary of test results for both the $\mathrm{OP}$ and Belfort gages were compiled for each site and are shown in tables 1 and 2. One data set was developed with non-event or zero data removed. Suspected false-positive data were removed to create another data set. An OP false positive was defined as a recorded response from the OP gage (typically 0.01) and a zero response from the Belfort gage when the site operator reported a zero precipitation day and there was no recorded opening of the NTN site sample collector. Paired t-tests were run on both the precipitation events and adjusted data sets, using a 95 percent confidence interval. Both data sets also were evaluated using the Wilcoxon signed-rank test (a non-parametric statistical test) to compare gage performance. The null hypothesis for the analysis was that the mean (paired t-test) or the median (Wilcoxon signed-rank test) difference between the gage measurements equal zero.
For the purpose of this study, all of the data recorded by the NovaLynx gages were used as a reference for comparing precipitation measurements from gages at sites IL11 and PA15 (table 3). No data sets were removed for these comparisons.

Table 1. Differences of precipitation measurements between the OTT PLUVIO Precipitation Gage and Belfort Universal Precipitation Gage 5-780, collected during phase III supplemental data evaluation

[Abbreviations: OP, OTT PLUVIO Precipitation Gage; Belfort, Belfort Universal Precipitation Gage 5-780.]

\begin{tabular}{|c|c|c|c|c|c|c|c|c|c|c|}
\hline \multirow[b]{2}{*}{ Site } & \multirow[b]{2}{*}{$\begin{array}{c}\text { Number } \\
\text { of days } \\
\text { of lost } \\
\text { data } \\
\text { OP/ } \\
\text { Belfort }\end{array}$} & \multicolumn{4}{|c|}{ Precipitation events dataset } & \multicolumn{5}{|c|}{ False positives removed dataset } \\
\hline & & $\begin{array}{l}\text { Number } \\
\text { of precip- } \\
\text { itation } \\
\text { events }\end{array}$ & $\begin{array}{l}\text { Mean } \\
\text { precip- } \\
\text { itation, } \\
\text { OP/ } \\
\text { Belfort } \\
\text { (inches) }\end{array}$ & $\begin{array}{l}\text { Median } \\
\text { precip- } \\
\text { itation, } \\
\text { OP/ } \\
\text { Belfort } \\
\text { (inches) }\end{array}$ & $\begin{array}{l}\text { Total } \\
\text { precip- } \\
\text { itation, } \\
\text { OP/ } \\
\text { Belfort } \\
\text { (inches) }\end{array}$ & $\begin{array}{l}\text { Number } \\
\text { of false } \\
\text { positives } \\
\text { removed }\end{array}$ & $\begin{array}{c}\text { Adjusted } \\
\text { number } \\
\text { of } \\
\text { events }\end{array}$ & $\begin{array}{l}\text { Adjusted } \\
\text { mean } \\
\text { precip- } \\
\text { itation, } \\
\text { OP/ } \\
\text { Belfort } \\
\text { (inches) }\end{array}$ & $\begin{array}{c}\text { Adjusted } \\
\text { median } \\
\text { precip- } \\
\text { itation, } \\
\text { OP/ } \\
\text { Belfort } \\
\text { (inches) }\end{array}$ & $\begin{array}{l}\text { Adjusted } \\
\text { total } \\
\text { precip- } \\
\text { itation, } \\
\text { OP/ } \\
\text { Belfort } \\
\text { (inches) }\end{array}$ \\
\hline $\begin{array}{l}\text { IL11 } \\
\text { East }\end{array}$ & $14 / 15$ & 66 & $0.26 / 0.25$ & $0.05 / 0.05$ & $16.97 / 16.46$ & 17 & 52 & $0.32 / 0.32$ & $0.10 / 0.11$ & $16.78 / 16.46$ \\
\hline $\begin{array}{l}\text { IL11 } \\
\text { West }\end{array}$ & $7 / 15$ & 109 & $0.18 / 0.17$ & $0.02 / 0.00$ & $19.23 / 18.10$ & 51 & 65 & $0.29 / 0.28$ & $0.06 / 0.06$ & $18.53 / 18.10$ \\
\hline MN16 & $7 / 21$ & 106 & $0.07 / 0.07$ & $0.01 / 0.01$ & 7.88/7.49 & 33 & 73 & $0.11 / 0.10$ & $0.03 / 0.03$ & $7.72 / 7.49$ \\
\hline NV03 & $11 / 12$ & 56 & $0.03 / 0.02$ & $0.01 / 0.00$ & $1.58 / 1.13$ & 34 & 22 & $0.06 / 0.05$ & $0.02 / 0.03$ & $1.24 / 1.13$ \\
\hline PA15 & $0 / 28$ & 75 & $0.24 / 0.25$ & $0.10 / 0.11$ & $17.75 / 18.41$ & 2 & 73 & $0.24 / 0.25$ & $0.13 / 0.12$ & $17.73 / 18.41$ \\
\hline
\end{tabular}

Table 2. Differences of measurements at the National Trends Network sites using the paired t-test and the Wilcoxon signed-rank test during phase III supplemental data evaluation

\begin{tabular}{|c|c|c|c|c|c|c|c|c|c|c|}
\hline \multirow{3}{*}{ Site } & \multicolumn{3}{|c|}{ Precipitation event data set } & \multicolumn{3}{|c|}{ False positive removed } & \multicolumn{2}{|c|}{$\begin{array}{l}\text { Precipitation event } \\
\text { data set }\end{array}$} & \multicolumn{2}{|c|}{$\begin{array}{l}\text { False positives } \\
\text { removed }\end{array}$} \\
\hline & $\begin{array}{c}\text { Mean } \\
\text { difference }\end{array}$ & $p$-value & $\begin{array}{c}\text { Mean } \\
\text { equals } \\
\text { zero }\end{array}$ & $\begin{array}{c}\text { Mean } \\
\text { difference }\end{array}$ & $p$-value & $\begin{array}{c}\text { Mean } \\
\text { equals } \\
\text { zero }\end{array}$ & $p$-value & $\begin{array}{c}\text { Median } \\
\text { equals } \\
\text { zero }\end{array}$ & $p$-value & $\begin{array}{c}\text { Median } \\
\text { equals } \\
\text { zero }\end{array}$ \\
\hline & \multicolumn{6}{|c|}{ Paired t-test } & \multicolumn{4}{|c|}{ Wilcoxon signed-rank test } \\
\hline $\begin{array}{l}\text { IL11 } \\
\text { East }\end{array}$ & 0.008 & 0.183 & $\begin{array}{l}\text { Do not } \\
\text { reject }\end{array}$ & 0.006 & 0.402 & $\begin{array}{l}\text { Do not } \\
\text { reject }\end{array}$ & 0.097 & $\begin{array}{l}\text { Do not } \\
\text { reject }\end{array}$ & 0.439 & $\begin{array}{l}\text { Do not } \\
\text { reject }\end{array}$ \\
\hline $\begin{array}{l}\text { IL11 } \\
\text { West }\end{array}$ & 0.010 & 0.001 & Reject & 0.007 & 0.205 & $\begin{array}{l}\text { Do not } \\
\text { reject }\end{array}$ & $\sim 0$ & Reject & 0.199 & $\begin{array}{l}\text { Do not } \\
\text { reject }\end{array}$ \\
\hline MN16 & 0.004 & 0.615 & $\begin{array}{l}\text { Do not } \\
\text { reject }\end{array}$ & 0.003 & 0.769 & $\begin{array}{l}\text { Do not } \\
\text { reject }\end{array}$ & 0.001 & Reject & 0.130 & $\begin{array}{l}\text { Do not } \\
\text { reject }\end{array}$ \\
\hline NV03 & 0.008 & $\sim 0$ & Reject & 0.005 & 0.373 & $\begin{array}{l}\text { Do not } \\
\text { reject }\end{array}$ & $\sim 0$ & Reject & 0.144 & $\begin{array}{l}\text { Do not } \\
\text { reject }\end{array}$ \\
\hline PA15 & -0.009 & 0.137 & $\begin{array}{l}\text { Do not } \\
\text { reject }\end{array}$ & -0.009 & 0.125 & $\begin{array}{l}\text { Do not } \\
\text { reject }\end{array}$ & 0.543 & $\begin{array}{l}\text { Do not } \\
\text { reject }\end{array}$ & 0.446 & $\begin{array}{l}\text { Do not } \\
\text { reject }\end{array}$ \\
\hline
\end{tabular}

Table 3. Differences of precipitation measurements between the Belfort Universal Precipitation Gage 5-780, OTT PLUVIO Precipitation Gage, and the NovaLynx Model 260-2510 Standard Rain and Snow Gage, using the paired t-test and Wilcoxon signedrank test at sites IL11, for 63 comparisons and PA15 for 64 comparisons.

[Abbreviations: --, no data; Belfort, Belfort Universal Precipitation Gage 5-780; OP, OTT PLUVIO precipitation gage; NovaLynx, NovaLynx Model 260-2510 Standard Rain and Snow Gage.]

\begin{tabular}{|c|c|c|c|c|c|c|c|c|}
\hline \multirow{2}{*}{$\begin{array}{l}\text { Gage } \\
\text { and } \\
\text { site }\end{array}$} & \multirow{2}{*}{$\begin{array}{l}\text { Mean } \\
\text { precip- } \\
\text { itation } \\
\text { (inches) }\end{array}$} & \multirow{2}{*}{$\begin{array}{c}\text { Median } \\
\text { precip- } \\
\text { itation } \\
\text { (inches) }\end{array}$} & \multirow{2}{*}{$\begin{array}{l}\text { Total } \\
\text { precip- } \\
\text { itation } \\
\text { (inches) }\end{array}$} & \multicolumn{3}{|c|}{ Paired t-test } & \multicolumn{2}{|c|}{$\begin{array}{l}\text { Wilcoxon signed- } \\
\text { rank test }\end{array}$} \\
\hline & & & & $\begin{array}{c}\text { Mean } \\
\text { difference }\end{array}$ & $p$-value & $\begin{array}{l}\text { Mean } \\
\text { difference } \\
\text { equals } \\
\text { zero }\end{array}$ & $p$-value & $\begin{array}{c}\text { Median } \\
\text { difference } \\
\text { equals } \\
\text { zero }\end{array}$ \\
\hline $\begin{array}{l}\text { Belfort, } \\
\text { IL11 }\end{array}$ & 0.28 & 0.06 & 17.33 & 0.037 & $\sim 0$ & Reject & $\sim 0$ & Reject \\
\hline $\begin{array}{l}\text { OP, } \\
\text { IL11 East }\end{array}$ & 0.33 & 0.07 & 20.93 & 0.305 & $\sim 0$ & Reject & 0.112 & $\begin{array}{l}\text { Do not } \\
\text { reject }\end{array}$ \\
\hline $\begin{array}{l}\text { OP, } \\
\text { IL11 West }\end{array}$ & 0.33 & 0.11 & 20.58 & -0.015 & 0.002 & Reject & 0.078 & $\begin{array}{l}\text { Do not } \\
\text { reject }\end{array}$ \\
\hline $\begin{array}{l}\text { NovaLynx, } \\
\text { IL11 }\end{array}$ & 0.32 & 0.08 & 19.66 & -- & -- & -- & -- & -- \\
\hline $\begin{array}{l}\text { Belfort, } \\
\text { PA15 }\end{array}$ & 0.37 & 0.19 & 23.94 & -0.011 & 0.026 & Reject & 0.003 & Reject \\
\hline $\begin{array}{l}\text { OP, } \\
\text { PA15 }\end{array}$ & 0.38 & 0.20 & 24.49 & -0.003 & 0.661 & $\begin{array}{l}\text { Do not } \\
\text { reject }\end{array}$ & 0.203 & $\begin{array}{l}\text { Do not } \\
\text { reject }\end{array}$ \\
\hline $\begin{array}{l}\text { NovaLynx, } \\
\text { PA15 }\end{array}$ & 0.38 & 0.20 & 24.69 & -- & -- & -- & -- & -- \\
\hline
\end{tabular}


The box plots in figure 1 show the differences in inches between the daily precipitation measured by the Belfort and OP precipitation gages. The median lines for the five sites are located at or close to zero, with slight variability of distribution for the middle 50 percent of the data. IL11 West and PA15 sites showed the greatest variability with the presence of several outliers. The site at PA15 had more outliers for positive differences indicating the Belfort gage recorded higher measurements than the OP gage.

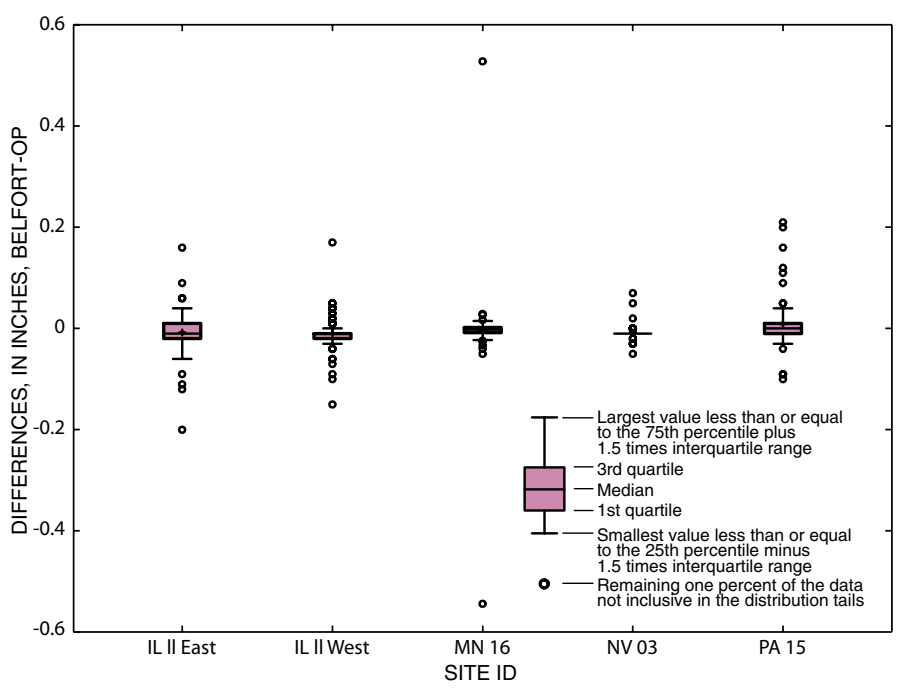

Figure 1. Differences between the daily precipitation measured by the Belfort Universal Precipitation Gage 5-780 and OTT PLUVIO precipitation gages.

Cumulative precipitation for all sites is shown in figure 2.

Throughout phase III supplemental testing, the precipitation events measured by the OP gages were generally higher than the Belfort gages. The gages at PA15 were the exception; cumulative precipitation at the Belfort gage was 0.68-in. higher than the OP gage. Paired t-tests showed no significant differences between the gage measurements at sites IL11 East, MN16, and PA15. When the false positives were removed, the measurements at all sites were not significantly different. Wilcoxon signed-rank tests showed that only the measurements at IL11 East and PA15 were not significantly different. When the false positives were removed, the analysis showed no significant difference between measurements at all sites.

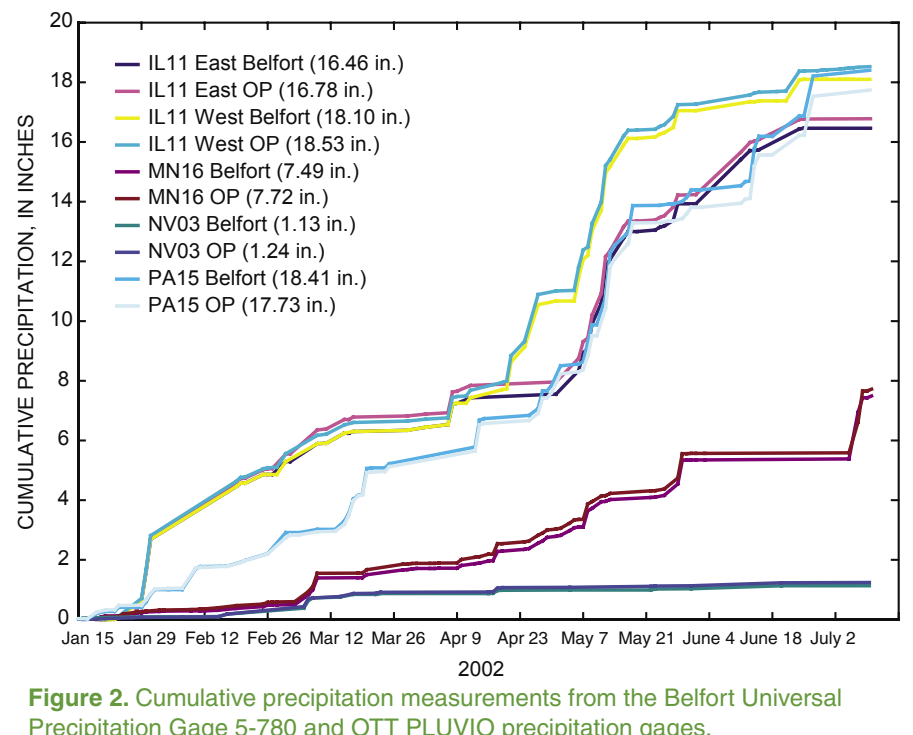

Figure 2. Cumulative precipitation measurements from the Belfort Universal Precipitation Gage 5-780 and OTT PLUVIO precipitation gages.
As part of the phase III supplemental testing analysis, precipitation measurements obtained at sites IL11 and PA15 by the Belfort and OP gages were compared to precipitation measurements recorded by the NovaLynx gages (table 3). No data sets were removed for these comparisons.

Cumulative precipitation measured at site IL11 for the Belfort gage was 17.33 in., the East OP gage was 20.93 in., the West OP gage was 20.58 in., and the NovaLynx gage measured 19.66 in. for 63 comparisons (fig. 3). Paired t-tests showed significant differences between the OP, Belfort, and NovaLynx gage measurements. Wilcoxon signed-rank tests showed no significant differences between the NovaLynx gage and both East and West OP gage measurements; however, the NovaLynx and the Belfort gage measurements were significantly different.

Cumulative precipitation measured at site PA15 for the Belfort gage was 23.94 in., the OP gage was 24.49 in., and the NovaLynx gage was 24.69 in. for 64 comparisons (fig. 3). Paired t-tests showed no significant difference between NovaLynx and OP gage measurements. Paired t-tests for the NovaLynx and the Belfort gage measurements were significantly different. The Wilcoxon signed-rank test showed no significant differences between the NovaLynx and OP gage measurements, but was significantly different for the NovaLynx and the Belfort gage measurements.

-Mary L. Tumbusch

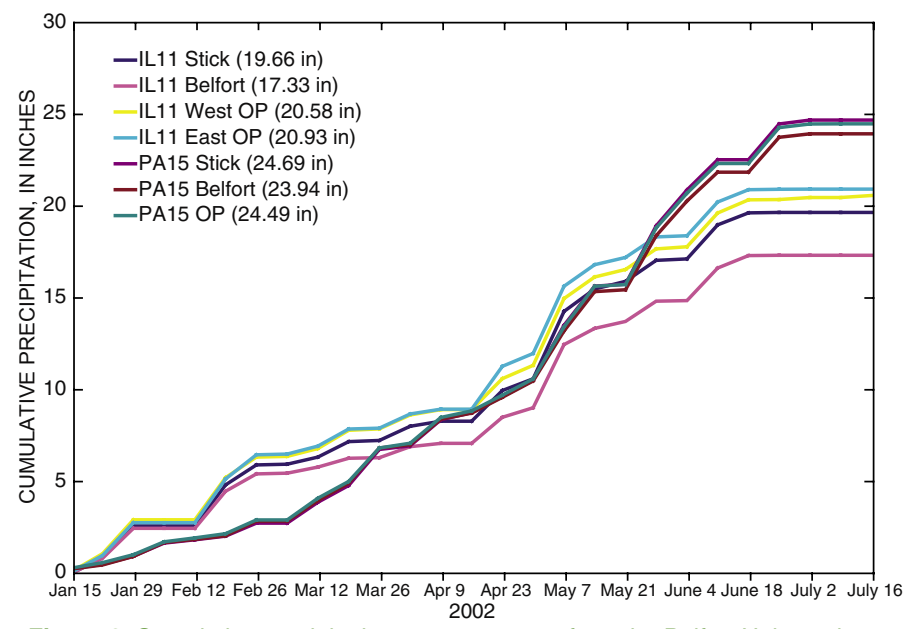

Figure 3. Cumulative precipitation measurements from the Belfort Universal Precipitation Gage 5-780, OTT PLUVIO precipitation gage, and NovaLynx Model 260-2510 Standard Rain and Snow Gage for the sites at IL11 East, IL11 West, and PA15.

\section{References}

National Atmospheric Deposition Program, 2003. NADP Site overview page, accessed March, 10, 2003 at URL http://nadp.sws.uiuc.edu/nadpoverview.asp.

Tumbusch, M.L., 2003, Evaluation of OTT PLUVIO Precipitation Gage versus Belfort Universal Precipitation Gage 5-780 for the National Atmospheric Deposition Program: U.S. Geological Survey Water-Resources Investigations Report 03-4167, 25 p. http://pubs.water.usgs.gov/wri034167.

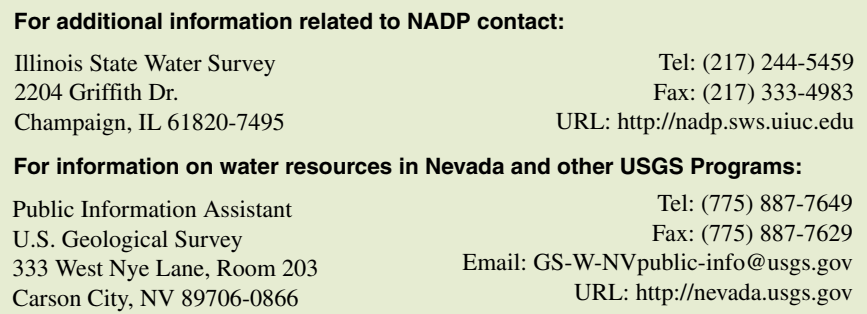

